\title{
Foraging competition, vigilance and group size in two species of gregarious antelope
}

\author{
Fredrik Dalerum ${ }^{1,2^{*}}$, Henrik Lange ${ }^{3}$, Christina Skarpe ${ }^{4}$, Tuulikki Rooke ${ }^{5}$, \\ Berit Inga ${ }^{5}$ \& Philip W. Bateman ${ }^{1}$ \\ ${ }^{1}$ Mammal Research Institute, Department of Zoology and Entomology, University of Pretoria, Pretoria, 0002 South Africa \\ ${ }^{2}$ Centre for Wildlife Management, University of Pretoria, Pretoria, 0002 South Africa \\ ${ }^{3}$ Institute of Neuroscience \& Centre for Behaviour and Evolution, Newcastle University, United Kingdom \\ ${ }^{4}$ Faculty of Forestry and Wildlife Management, Hedmark University College, NO-2480 Elverum, Norway \\ ${ }^{5}$ Department of Wildlife, Fish and Environmental Sciences, Swedish University of Agricultural Sciences, Umeå, Sweden
}

Received 7 January 2008. Accepted 4 June 2008

\begin{abstract}
In gregarious species, rates of foraging behaviour are often positively related to group size while there is simultaneously a negative relation between group size and vigilance. Although the mechanisms underlying these behavioural patterns are still incompletely understood, decreased predation risk or increased foraging competition in larger groups have been put forward as two possible explanations. Since most empirical tests of these two hypotheses have used manipulative experiments, they have mainly been limited to small-bodied species or to animals in captivity. Here we suggest a time-budget model to test for the causal effects of predation risk and foraging competition that does not necessitate manipulative approaches. We used this method on two species of gregarious antelope, blesbok (Damaliscus pygargus phillipsi) and impala (Aepyceros melampus). We suggest that increased foraging competition in large groups drives the negative relationship between vigilance and group size in these species. This study shows that observational data can be used to test explicit hypotheses on species that are logistically unsuitable for manipulative experiments, and also render support for hypotheses suggesting that factors unrelated to predation risk are involved in shaping the differences in vigilance rates over different group sizes in gregarious species.
\end{abstract}

Key words: foraging competition, anti-predatory vigilance, group size effect, observational data, predation risk.

\section{INTRODUCTION}

A positive relationship is often found between rates of foraging behaviour and group size combined with a negative relationship between group size and vigilance (reviews in Elgar 1989; Lima \& Dill 1990; Roberts 1996; Beauchamp 2003). An oftensuggested interpretation is that increased foraging is a consequence of a decreased pressure to be vigilant: animals in larger groups can allocate more time to foraging-related behaviour (Roberts 1996; Beauchamp 2003). An opposing interpretation is that intra-group foraging competition would cause an increased rate of foraging behaviour in large groups, and an equivalent decrease in time spent in other behaviour patterns, including vigilance (Elgar 1989; Blumstein et al. 2001; Beauchamp \& Ruxton 2003; Fortin et al. 2004a,b).

There is still no general consensus regarding

\footnotetext{
*To whom correspondence should be addressed.
}

E-mail: fredrik.dalerum@zoology.up.ac.za which of these two explanations might better explain the observed correlations between group size and rates of vigilance and foraging behaviour (Beauchamp 2001; Roberts 2003). It has been suggested that the most fruitful way to address the issue is to conduct controlled experiments (Beauchamp 2003; Bednekoff 2003). While this is true, it may be argued that limiting vigilance studies to seed-feeding bird species or mammals in enclosures may affect our understanding of these processes. In some species (e.g. grazing herbivores) it is logistically daunting to conduct naturalscale controlled experiments, but since group-size effects are common in such species (e.g. Burger \& Gochfield 1994; Matson et al. 2005) there is still a need for non-experimental methods that enable us to study them. However, observations limited to patterns of vigilance and rates of foraging behaviour would not allow us to distinguish between the two above-mentioned explanations 
for the group size effect (Treves 2000).

Instead, we suggest that these patterns should be analysed together with observations on accompanying changes in behaviours that are not directly related to foraging or vigilance. For instance, if there is less need to be vigilant, one might expect individuals to engage more in behaviour such as grooming as well as in more foraging. Equivalently, if there is an increased need to forage, one might expect individuals to not only allocate less time to being vigilant, but also to other behaviour(s) not related to foraging or vigilance. This would lead to a situation where we can make three different predictions regarding the accompanying changes in behaviour not related to foraging or vigilance over increasing group sizes: i) decreased vigilance rates lead to an increase in all other behaviour, suggesting a causal effect of vigilance rates on the observed patterns (Fig. 1a); ii) increased rates of foraging behaviour lead to a decrease in all other behaviour, suggesting a causal effect of foraging rates (Fig. 1b), and iii) only vigilance and rates of foraging behaviour are affected by group size (Fig. 1c). This last pattern is symmetric and hence does not let us distinguish between possible causal effects of foraging or vigilance. Another shortcoming with our approach is that many species may have behavioural overlap, i.e. they may be able to forage while being vigilant or they may be vigilant while doing something else, for instance grooming (Caro 2005). However, such problems may possibly be reconciled through a careful definition of behavioural categories when collecting data.

We tested these predictions using data from two species of gregarious ungulate: blesbok (Damaliscus pygargus phillipsi) and impala (Aepyceros melampus). Blesbok are diurnal grazers that form mixed-sex or bachelor groups of typically 10-25 individuals, although they may congregate into much larger groups, particularly in areas with a post-burn flush of new grass (Skinner \& Chimimba 2005). Impala are diurnal mixed-feeders (primarily grazers) that are usually found in loosely associated female or mixed sex groups of 6-20 animals, but they may also congregate into larger groups of over 100 (Skinner \& Chimimba 2005). Solitary territorial males and bachelor groups do also occur during parts of the year.

\section{METHODS}

We conducted observations on groups of blesbok at Rietvlei Nature Reserve, Gauteng Province, South Africa during October 2006. This reserve

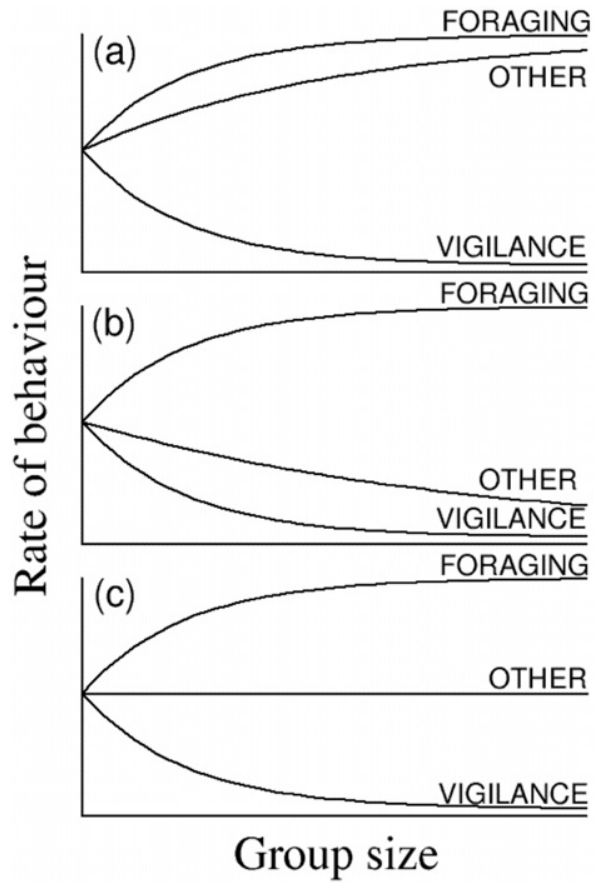

Fig. 1. Visualizations of conceptual time budget models for the relations between group size and rates of behaviour for three possible strategies of behavioural trade-offs at different group sizes: a, decreased vigilance in large groups is compensated for by an overall increase in all other behaviour (i.e. vigilance rates drives behavioural changes over varying group sizes); $\mathbf{b}$, increased rates of foraging behaviour is compensated for by an overall decrease in all other behaviour (i.e. rates of foraging behaviour drives behavioural changes over varying group sizes); c, only vigilance and rates of foraging behaviour are affected by group size (does not distinguish between possible causal effects of foraging or vigilance).

supports cheetah (Acinonyx jubatus) and blackbacked jackal (Canis mesomelas), both predators that can prey on new-born blesbok. We collected scan data on six discrete groups of blesbok ranging from 10 to 40 individuals. Duration of scan observations ranged from 30 to 60 minutes. Observation periods were ended when the group moved out of visible range or the group was regarded to have stopped foraging as explained below. Every 10 minutes, the whole group was scanned and the numbers of animals foraging, being vigilant, or carrying out other activities (walking, grooming, and social interactions) were recorded. Animals standing up with their heads below shoulder height and the muzzle down towards the ground were regarded as foraging even if they simultaneously were conducting other behaviour (e.g. walking), 
whereas animals with their heads raised above shoulder level and scanning their surroundings were considered to be vigilant even if conducting other behaviour. Animals were regarded as vigilant only when standing up, and any observations on groups with large numbers of animals lying down where terminated to avoid the confounding problem of animals simultaneously being vigilant while ruminating (see below). Hence, behaviour was only scored as 'other' (i.e. not foraging or vigilance) if it clearly did not consist of any element of either foraging or vigilance behaviour.

In addition to scan observations, we conducted focal observations on 39 individuals from 14 different groups, ranging in size from lone individuals up to 55 animals. Focal observations were conducted for 10-minute periods, and we recorded time spent in the behavioural categories defined above. As many animals as possible were focal-observed in each group until the group was out of sight. If group composition changed during a focal-sampling period, that sample was terminated. However, we did sample four groups over more than one group size. Animals were chosen at random, and we were often able to sample the whole group, especially for small group sizes. We only conducted one focal period per animal.

We made behavioural observations only during active foraging time in the mornings (between 05:00 and 08:00). We stopped recordings when more than half of the observed group were lying down and not actively grazing to avoid behaviour such as ruminating adversely affecting our results. Animals generally settled down to ruminate fairly rapidly and simultaneously, so that we do not believe that time of observation substantially affected the observed behavioural patterns. Both scan and focal observations were made from cars at distances of 50 to 400 metres.

We conducted behavioural observations on impala groups on the open floodplains at the Chobe riverfront, Botswana. This area supports lion (Panthera leo), leopard (Panthera pardus), African wild dog (Lycaon pictus) and spotted hyaena (Crocuta crocuta). For impala we conducted only scan observations because of time constraints. Data were collected as described above except that groups were scanned every five minutes for a 30-minute period. We conducted scan observations on 10 groups ranging from 10 to 110 individuals. Numbers of scans per group ranged from three to seven. Observations were made during two periods in 2001:25-26 August and 25 Novem- ber to 1 December. Three groups were observed in the first observation period and seven in the second. Only adult individuals were included in our group-size estimates, although lambs were present in all groups observed during the second observation period.

We used mixed generalized linear models with a logit link function and a binomial error structure to analyse the effects of group size on number of animals engaged in different behaviour types during scan observations. We used the raw number of animals engaged/not engaged in a behaviour type as a binary response variable, group size as a fixed continuous variable and group identity as a repeated measures (random) factor. We fitted one model for each species and behavioural category. For the models on impala, we also included observation period as a fixed factor. Since we did multiple tests of the beta coefficients, we have adjusted the probabilities for the coefficient tests for multiple comparisons using the false discovery rate method suggested by Benjamini \& Hochberg (1995). We used the absolute values of the beta coefficients as a measure of the relative strength of the effects of group size on foraging, vigilance and other behaviour. Although such comparisons generally are done with standardized coefficients (Selvin 1998), raw absolute values are appropriate in our case since they were derived from an independent variable with the same unit of measure (i.e. group size) for all three behavioural categories.

We used mixed linear models to test for the effect of group size on time engaged in different behaviour during focal observations. In the models we included only animals sampled for a full 10-minute focal period, and used time in seconds as response variable, group size as a fixed continuous variable and animal nested within group as a random factor to avoid pseudoreplication. For the models of time spent vigilant and time spent neither foraging nor vigilant, we log-transformed time engaged in behaviour to improve the model fit. As in our analyses of scan data, we adjusted the probabilities for our beta coefficient tests for multiple comparisons.

Statistical analyses were carried out using the statistical package R version 2.4.0 for Linux (http:// www.r-project.org).

\section{RESULTS}

Scan data did not reveal a significant group-size effect on rate of foraging behaviour within groups of blesbok ( $\left.\beta=0.01, z=-1.586, P_{\text {adjusted }}=0.11\right)$, but showed a significant group-size effect on rate 


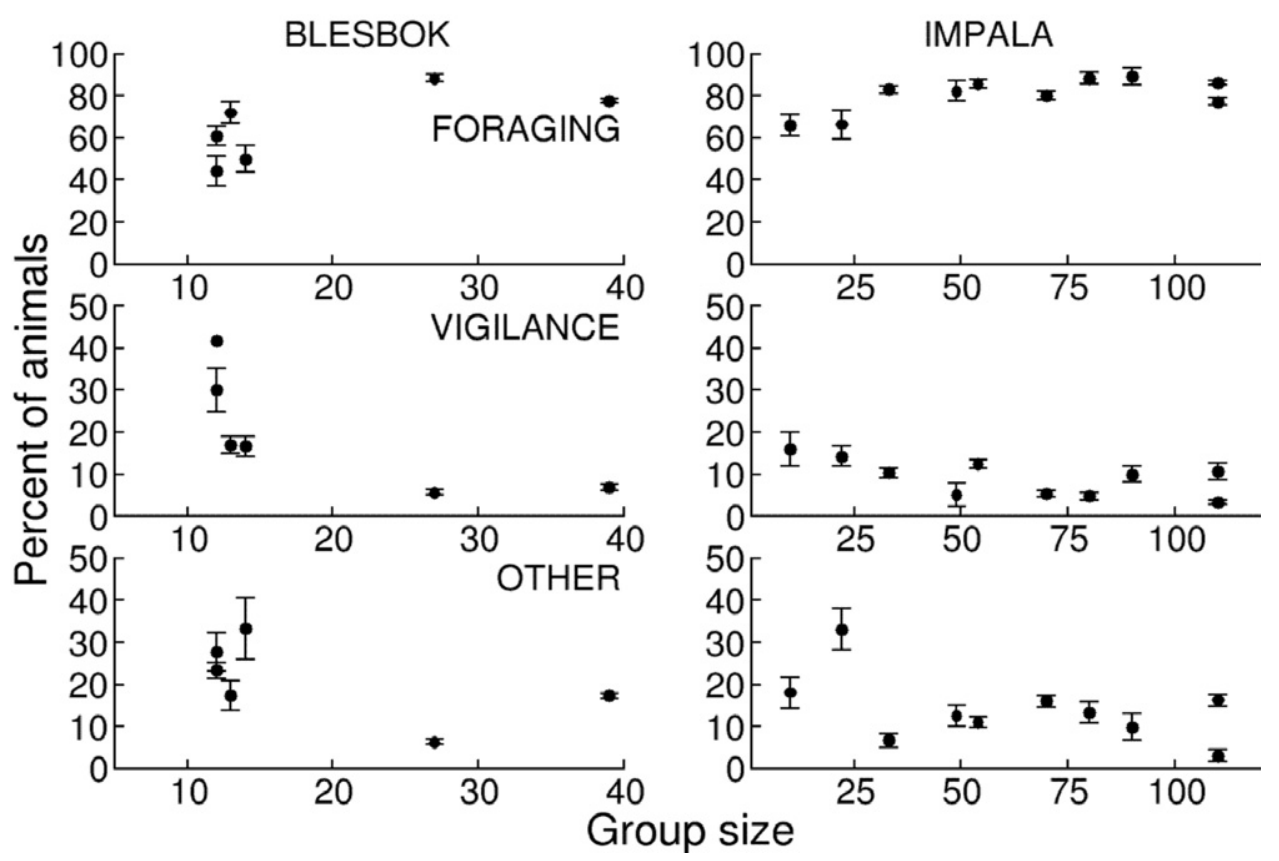

Fig. 2. Patterns of behavioural change over different group sizes in six blesbok and 10 impala groups, presented as mean and S.E. percentage of animals engaged in foraging, vigilance and behaviours not related to foraging or vigilance during scan observations.

of foraging behaviour within groups of impala $(\beta=$ $0.006, \mathrm{SE}_{\beta}=0.002, \mathrm{z}=2.606, P_{\text {adjusted }}=0.01$ ) (Fig. 2). Scan data showed significant negative group-size effects on vigilance rates within groups of both blesbok $\left(\beta=-0.03, \mathrm{SE}_{\beta}=0.01, \mathrm{z}=-2.375\right.$, $\left.P_{\text {adjusted }}=0.05\right)$ and impala $\left(\beta=-0.01, \mathrm{SE}_{\beta}=0.003\right.$, $\left.\mathrm{z}=-3.769, P_{\text {adiusted }}<0.01\right)$. Furthermore, scan data showed negative group-size effects on rate of behaviour not related to foraging or vigilance for both blesbok $\left(\beta 0.03, \mathrm{SE}_{\beta}=0.01 \mathrm{z}=-2.121, P_{\mathrm{ad}-}\right.$ $=0.05)$ and impala $\left(\beta=0.005, \mathrm{SE}_{\beta}=0.002, \mathrm{z}=\right.$ $-2.165, P_{\text {adiusted }}=0.03$ ) (Fig. 2). Observation period did not affect the number of animals foraging $\left(\chi^{2}=\right.$ 0.03 , d.f. $=1, P=0.85$ ), the number of animals being vigilant $\left(\alpha^{2}=0.57\right.$, d.f. $\left.=1, P=0.47\right)$ or the number of animals neither foraging nor vigilant $\left(\chi^{2}=0.20\right.$, d.f. $\left.=1, P=0.65\right)$ in impala.

The absolute values of the beta coefficients indicated that blesbok group size had a stronger effect on vigilance $(|\beta|=0.03)$ and behaviour not related to foraging or vigilance $(|\beta|=0.03)$ than on foraging behaviour $(|\beta|=0.01)$. For impala on the other hand, group size had a stronger effect on behaviour not related to foraging or vigilance $(|\beta|=$ $0.05)$ than on either foraging $(|\beta|==0.02)$ or vigilance $(|\beta|=0.01)$. Focal observations on blesbok did not reveal a group-size effect, on either rates of foraging behaviour $\left(\beta=-0.25, t_{22}=0.152\right.$, $\left.P_{\text {adiusted }}=0.88\right)$, or vigilance rates $\left(\beta=0.03, t_{22}=\right.$ $1.264, P_{\text {adjusted }}=0.33$ ) or rates of behaviour not related to foraging or vigilance $\left(\beta=0.03, t_{22}=1.464\right.$, $\left.P_{\text {adjusted }}=0.133\right)$ (Fig. 3).

During both scan and focal observations, behaviour not related to vigilance or foraging mainly consisted of walking (while not foraging or being vigilant at the same time) (observed in $53.6 \%$ of the scan events on blesbok and in $84.6 \%$ of the scan events on impala, and constituted $0-32 \%$ of focal time in blesbok), but also of grooming (observed in $21.6 \%$ of the scan events of blesbok and constituted $0-44.8 \%$ of focal time) and lying down (observed in $21.6 \%$ of the scan events of blesbok).

\section{DISCUSSION}

Scan data on both blesbok and impala showed a negative relation between vigilance and group size, but showed a positive relationship between group size and rates of foraging behaviour only for impala. This supports the generally observed 'group size' effect in impala (Elgar 1989; Matson et al. 2005), whereas our data for blesbok are less clear. A lack of group-size effects has also been reported in other recent studies (Treves 2000), 


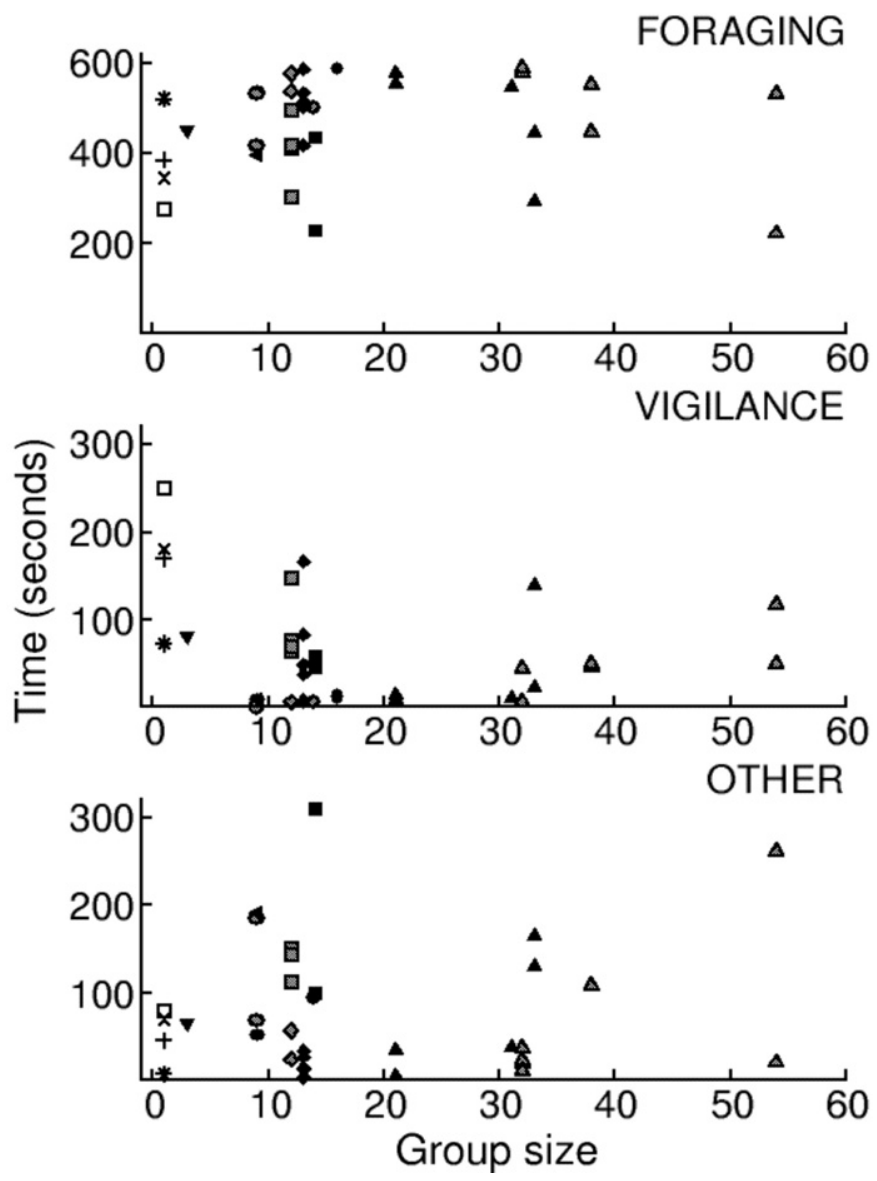

Fig. 3. Patterns of behavioural change over different group sizes in 39 individual blesbok, presented as time (seconds) spent engaged in foraging, vigilance and behaviours not related to foraging or vigilance during 10-minute focal observations. Data are from 14 different groups, and each data point is the raw time for each individual. The different symbols are coding for each blesbok group. Note the different scales on the $y$-axes between behavioural categories.

suggesting that relationships between group size, foraging and vigilance can be highly variable between species. Our scan data showed a negative relationship between behaviour not related to foraging or vigilance and group size for both blesbok and impala. This conforms to patterns related to a causal effect of increased rates of foraging behaviour for impala (i.e. Fig. 1b), and thus supports the prediction that increasing foraging rates in larger groups drive the negative group-size effect on vigilance rates for this species.

Our model assumes that either foraging or vigilance behaviour drives behavioural changes over different group sizes. Another possibility is that behaviour not related to foraging and vigilance is the driving force behind changes in foraging and vigilance rates. This could potentially lead to an additional number of behavioural patterns. For instance, an increase in non-foraging/non-vigilance behaviour with increasing group size could lead to three patterns of change in vigilance and foraging rates: i) a decrease in both vigilance and foraging, ii) a decrease in foraging but not in vigilance, iii) a decrease in vigilance but not in foraging. Conversely, a decrease in non-foraging/nonvigilance behaviour with increasing group size could lead to: i) an increase in both vigilance and foraging, ii) an increase in foraging but not in vigilance, iii) an increase in vigilance but not in foraging. None of these patterns fit the data for blesbok, since both vigilance and non-foraging/ non-vigilance decline with increasing group size. Furthermore, while Elgar (1989) suggested that group size effects may arise from larger groups foraging in areas of different food abundance compared to smaller groups, such confounding 
effects are likely to be most pronounced in species which rely on very patchy resources. In grazing or mixed feeding ungulates, this is rarely the case. We therefore suggest that the most parsimonious explanation for our observed patterns in blesbok is that increased foraging rates in larger groups may drive the negative group-size effect on vigilance rates for this species as well, but that we failed to detect such an effect with our observational data.

This interpretation agrees with the relative strength of the effect that group size had on foraging, vigilance and behaviour not related to foraging or vigilance, where both vigilance and behaviour not related to foraging or vigilance were equally affected by group size in blesbok, whereas there was no effect on foraging. In impala, group size affected behaviour not related to foraging or vigilance substantially more than either foraging or vigilance, but affected foraging more than vigilance.

Our model assumes no behavioural overlap, so that non-foraging and non-vigilance behaviour is unrelated to foraging or vigilance. In our case, most of the 'other' behaviour recorded consisted of walking. We took careful consideration when collecting data to identify any overlapping behaviour between walking and foraging or vigilance. We recorded any such overlap simply as being foraging or being vigilant regardless of which other behaviour the animal might have been engaged in simultaneously. Although we may have missed some levels of behavioural overlap, we believe that most of our 'other' behaviour was not directly related to vigilance or foraging, thus meeting the assumption behind the model.

We suggest that the observed decrease in vigilance rates in large groups was caused by an increase in foraging competition. Although grasslands and savannas such as the ones found in southern Africa appear homogeneous and without much scope for generating interference competition, recent work has shown that individuals of grazing species might experience a certain level of direct interference competition through patch depletion (Rowcliffe et al. 2004). On several occasions we observed grazing blesbok having their muzzle pushed aside by another group member, indicating a combination of scramble and interference competition within the ungulate groups.

Both blesbok and impala are gregarious grazers, and generally forage in loosely connected fission/ fusion groups (Murray 1981; Skinner \& Chimimba 2005). Such group dynamics have been suggested to affect the effect of group size on foraging rates, with a predicted decrease in foraging rates in larger groups when the group size is dynamic (Bednekoff \& Lima 2004). However, group size has been reported as directly affecting foraging decisions in browsing impala (Fritz \& De GarineWichatitsky 1996), suggesting that both foraging competition and foraging rates may indeed increase with increasing group size in this species. Blesbok generally feed on lower-quality food than impala (Klein \& Fairall 1986), and this difference in foraging ecology might explain the differences in the effect of group size on foraging rates in our study.

We found discrepancies in the effects of group size on behaviour not only between species but also between scan and focal data. Most studies exploring the relationships between vigilance rates and group size have used focal data, since the evolutionary theory behind any relationship between behaviour and group size is focused on individual behaviour rates (e.g. Pulliam 1973). Scan observations may, however, in some cases be better suited to test predictions related to group-size effects since they effectively will represent the average of types of behaviour of individuals in the group over the observation period (Altman 1974; Martin \& Bateson 1986). Focal data may be greatly influenced by, for instance, position in the group of the focal animal (since animals at the edge of a group often have higher vigilance rates than animals at the centre of a group) and could therefore be prone to observer bias. This will be particularly pronounced if the number of observed animals is small and if the focal observation period is short relative to the time it takes for animals to shift positions within a group. If group position is dynamic, so that all individuals spend on average an equal time at the edge, average behaviour rates within a group should be directly related to individual behaviour rates. Scan data can in such situation be less prone to observer bias than focal data, and may provide more accurate measurement of differences in behavioural rates over different group sizes. We suggest that a low number of observed individuals using focal observations coupled with factors not monitored by our behavioural protocol caused the discrepancies between scan and focal data with regard to detected differences of behaviour over varying group sizes.

This study highlights that explicit hypotheses regarding mechanisms generating behavioural 
patterns can be tested without manipulative experiments, but that the interpretation of field data from such tests can be difficult. Although experimental work is essential to test developed theory, many organisms are not well suited for such manipulations. Yet, for theory to be broadly applicable it needs to be tested on a wide range of organisms, not exclusively those that are logistically suited for manipulative experiments. We have suggested a conceptual model for using observational data to test explicit hypotheses regarding the mechanisms generating the often-observed group-size effects on rates of foraging behaviour and vigilance. We suggest that this and similar models are further developed to improve our understanding of behavioural mechanisms especially in species that are daunting to manipulate experimentally. We particularly encourage further studies disentangling the effect of group size on different components of foraging behaviour in grazing antelope, since this information will be needed to fully understand the role of competition in shaping the relationships between group size and rates of behaviour.

\section{CONCLUSION}

We suggest a time-budget model to test for the causal effects of predation risk and foraging competition on the often-observed group-size effects on vigilance and foraging rates that does not necessitate manipulative experiments. Observational scan data on two gregarious species of antelope provided contrasting resolution in the results regarding the causal mechanisms behind observed patterns of behavioural change over different group sizes. Data on impala supported predictions suggesting that increased rates of foraging behaviour in larger groups drives the negative relationship between vigilance and group size, whereas data on blesbok provided less clear results. However, since we found a negative group-size effect on vigilance coupled with a negative group-size effect on behaviour not related to foraging or vigilance, we suggest that these relationships were also caused by an increase in foraging rates in blesbok but that such an increase remained undetected by our observations. We propose that these patterns were caused by increased foraging competition. Our data generally support recent studies highlighting the importance of factors not directly associated with predation risk for the negative relation between vigilance and group size.

\section{ACKNOWLEDGEMENTS}

The city of Tshwane kindly gave permission to work in Rietvlei Nature Reserve. We are also grateful to the Department of Wildlife and National Parks, Botswana, for the generous permission (WP/Nat I (79)) to visit Chobe National Park and to the BONIC project for being our host during the stay in Chobe. Kim Leighton and Lydia Belton provided invaluable assistance with behavioural observations on blesbok. Adrian Shrader, Gilbert Roberts, Peter Jarman and an anonymous reviewer gave valuable comments on the manuscript.

\section{REFERENCES}

ALTMANN, J. 1974. Observational study of behavioural sampling methods. Behaviour 49: 227-265.

BEAUCHAMP, G. 2001. Should vigilance increase with increasing group size? Behav. Ecol. Sociobiol. 51: 47-52.

BEAUCHAMP, G. 2003. Group-size effect on vigilance: a search for mechanisms. Behav. Proc. 63: 111-121.

BEAUCHAMP, G. \& RUXTON, G.D. 2003. Changes in vigilance with group size under foraging competition. Am. Nat. 161: 672-675.

BEDNEKOFF, P.A. 2003. Testing explanations of the group size effect on vigilance: let's be direct. Behav. Proc. 63: 135-136.

BEDNEKOFF, P.A. \& LIMA, S.L. 2004. Risk allocation and competition in foraging groups: reversed effects of competition if group size varies under the risk of predation. Proc. Roy. Soc. B 271: 1491-1496.

BENJAMINI, Y. \& HOCHBERG, Y. 1995. Controlling the false discovery rate: a practical and powerful approach to multiple testing. J. Roy. Stat. Soc. B 57: 289-300.

BLUMSTEIN, D.T., DANIEL, J.C. \& EVANS, C.S. 2001. Yellow-footed rock wallaby group size effects reflect a trade-off. Ethology 107: 655-664.

BURGER, J. \& GOCHFELD, M. 1994. Vigilance in African mammals: differences among mothers, other females, and males. Behaviour 131: 153-169.

CARO, T. 2005. Antipredator defences in birds and mammals. University of Chicago Press, Chicago.

ELGAR, M.A. 1989. Predator vigilance and group size in mammals and birds: a critical review of empirical evidence. Biol. Rev. 64: 13-33.

FRITZ, H. \& DE GARINE-WICHATITSKY, M. 1996. Foraging in a social antelope: effects of group size on foraging choices and resource perception in impala. J. Anim. Ecol. 65: 736-742.

FORTIN, D., BOYCE, M. \& MERRYLL, E.H. 2004a. Multi-tasking by mammalian herbivores: overlapping processes during foraging. Ecology 85: 2312-2322.

FORTIN, D., BOYCE, M. \& MERRYLL, E.H. 2004b. Foraging constraints on vigilance in mammalian herbivores. Oikos 107: 172-180.

KLEIN, D.R. \& FAIRALL, N. 1986. Comperative foraging behaviour and associated energetics in impala and blesbok. J. Appl. Ecol. 23: 489-502.

LIMA, S.L. \& DILL, L.M. 1990. Behavioural decisions made under the risk of predation: a review and 
prospectus. Can. J. Zool. 68: 619-640.

MARTIN, P.C. \& BATESON, P. 1986. Measuring behaviour. Cambridge University Press, Cambridge.

MATSON, T.K., GOLDIZEN, A.W. \& PUTLAND, D.A. 2005. Factors affecting the vigilance and flight behaviour of impalas. S. Afr. J. Wildl. Res. 35: 1-11.

MURRAY, M.G. 1981. Structure of associations in impala Aepyceros melampus. Behav. Ecol. Sociobiol. 9: 23-33.

PULLIAM, H.R. 1973. On the advantages of flocking. J. Theoret. Biol. 38: 419-422.

ROBERTS, G. 1996. Why individual vigilance decreases as group size increases. Anim. Behav. 51: 10771086.
ROBERTS, G. 2003. The group-size effect in nonfeeding animals. Behav. Proc. 63: 127-128.

ROWCLIFFE, J.M., PETTIFOR, R.A. \& CARBONE, C. 2004. Foraging inequalities in large groups: quantifying depletion experienced by individuals in goose flocks. J. Anim. Ecol. 73: 97-108.

SELVIN, S. 1998. Modern applied biostatistical methods. Oxford University Press, Oxford.

SKINNER, J.D. \& CHIMIMBA, C.T. 2005. The mammals of the southern African subregion. Cambridge University Press, Cambridge.

TREVES, A. 2000. Theory and methods in studying vigilance and aggregation. Anim. Behav. 60: 711-722. 